\title{
Penile Metastatic Disease Presenting as Malignant Priapism: A Case Report
}

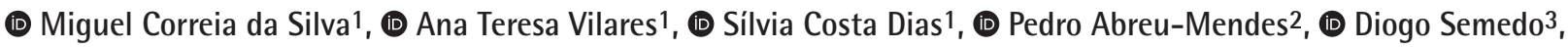 \\ (D) Raquel Portuga|3, (D) António J. Madureira1 \\ ${ }^{1}$ Centro Hospitalar Universitário de São João, Department of Radiology, Porto, Portugal \\ ${ }^{2}$ Centro Hospitalar Universitário de São João, Department of Urology, Porto, Portugal \\ ${ }^{3}$ Centro Hospitalar Universitário de São João, Department of Pathology, Porto, Portugal
}

\section{Abstract}

Penile metastatic disease is an uncommon condition with a dismal prognosis. Reported herein is a case of metachronous penile metastasis from bladder urothelial carcinoma, which presents as malignant priapism without tumoral masses. The authors explain the imaging and pathologic findings that led to its diagnosis and staging.

Keywords: Penile metastasis, malignant priapism, bladder urothelial carcinoma, tumoral emboli

\section{Introduction}

Penile metastasis is a relatively rare condition, with only approximately 500 cases published since first reported in 1870 (1). The most common primary sites associated with metastatic penile disease include urogenital (70\%) and gastrointestinal tumors (21\%) (2), especially of pelvic origin.

Clinical manifestations vary widely, including penile nodules or masses, skin lesions, malignant priapism, and less commonly, hematuria or lower urinary tract symptoms. These lesions are often associated with disseminated disease and predict a poor prognosis (3)

Reported herein is a case of metachronous penile metastasis from bladder urothelial carcinoma, which presents as priapism.

\section{Case Reports}

Reported herein is a case of a 79-year-old male, who presented to the emergency department of our institution with persistent vomiting due to intestinal obstruction, secondary to adhesions. Additionally, the patient reported a 2-week history of painful and inconstant erection that did not respond to medical therapy.
The patient was diagnosed with prostatic adenocarcinoma (Gleason 6) 15 years ago. Three years before the admission, the simultaneous transurethral resection of the prostate and bladder showed not only prostate tumoral persistence but also a papillary urothelial carcinoma. Radical cystoprostatectomy was performed. The pathological evaluation of the specimen revealed a high-grade invasive bladder urothelial carcinomapT4aN2R0 and concomitant prostate acinar adenocarcinoma, Gleason 9 (5+4)-pT3aN0R1.

On physical examination, despite abdominal pain, a rigid penile shaft was found, with tenderness on palpation and no significant hypoesthesia. No palpable nodules and no overlying penile skin lesions were found.

The ultrasound revealed an edematous thickening of the overlying skin and a few areas of heterogeneous echotexture in both corpora cavernosa. Color Doppler evaluation was unremarkable.

The subsequent magnetic resonant evaluation revealed patchy ill-defined areas of decreased T2-signal intensity along with both corpora cavernosa, which lacked enhancement after gadolinium administration, thus suspicious for tumoral invasion/ microthrombosis (Figure 1).

Correspondence: Miguel Correia da Silva MD, Centro Hospitalar Universitário de São João, Department of Radiology, Porto, Portugal

Phone: +351 225095539 E-mail: miguel.ncds@gmail.com ORCID-ID: orcid.org/0000-0001-5392-2808

Received: 31.03 .2021

Accepted: 17.04.2021

Cite this article as: Silva MC, Vilares AT, Dias SC, Abreu-Mendes P, Semedo D, Portugal R, Madureira AJ. Penile Metastatic Disease Presenting as Malignant

Priapism: A Case Report. J Urol Surg 2021;8(4):297-299.

${ }^{\circledR}$ Copyright 2021 by the Association of Urological Surgery / Journal of Urological Surgery published by Galenos Publishing House. 
The patient underwent a biopsy of the left corpus cavernosum that showed tumoral emboli from bladder urothelial carcinoma and thrombosis in the cavernosal spaces, which was contiguous with the neoplasia (Figure 2).

Multiple diffuse skeletal metastases were found on bone scintigraphy.

The case was presented to the multidisciplinary uro-oncology team of our institution that suggested palliative immunotherapy with pembrolizumab.

One month after the above-mentioned events, the patient died from tumor lysis syndrome.

\section{Discussion}

Penile metastatic cancer is an uncommon disease with a dismal prognosis.

Most cases arise from the genitourinary origin, with bladder cancer equaling prostate cancer ( $30 \%$ of cases) according to Zhang et al. (2) comprehensive review.

Despite rich penile blood supply, the mechanism that can explain the rarity of this secondary involvement is unclearly known. A few mechanisms have been hypothesized, which include direct tumor extension, vascular spread, or iatrogenic implantation $(4,5)$.

The most common scenario according to the literature on its clinical presentation is a tumoral mass appearance, usually in the shaft (6). Malignant priapism is reported in $20-53 \%$ of cases (4); therefore, the appearance of this sign in patients with a history of malignancy, especially of pelvic origin, should warrant the exclusion of a metastatic cause. However, unlike our case, priapism is rarely the first manifestation of disease with no accompanying tumoral mass. This phenomenon is mainly caused by tumoral invasion of the corpora cavernosa, vein occlusion, or interference with neural pathways, which predicts an even poorer prognosis (3).

To evaluate patients who are suspected of penile metastasis, a histopathologic examination is needed for a definitive diagnosis. Many imaging modalities play a role in the management and staging evaluation of these cases. Ultrasound is helpful in the early diagnosis of masses and Doppler ultrasonography in differentiating high-flow from low-flow priapism. Magnetic resonance imaging provides superior soft-tissue contrast and spatial resolution and therefore, can more accurately evaluate tumor size and anatomic structures invasion. Computed tomography (CT) and positron emission tomography/CT are relevant in the assessment of nodal and distant metastasis.

The differential diagnosis includes primary penile lesions (malignant, premalignant, or inflammatory) and in cases of priapism, other causes should be excluded, such as hematologic, trauma, iatrogenic, or drug-induced.

Treatment depends on the overall performance status but is frequently palliative, for symptom relief. Some patients may

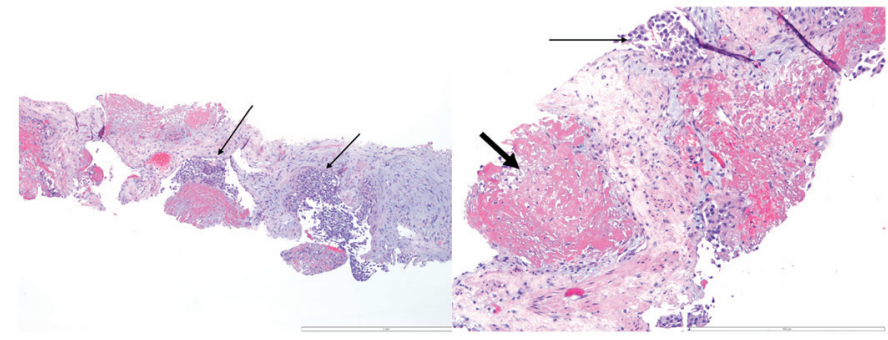

Figure 2. Corpus cavernosum biopsy with tumoral emboli from bladder urothelial carcinoma (thin arrows) and thrombosis in the cavernosal spaces that is contiguous with the neoplasia (thick arrow)
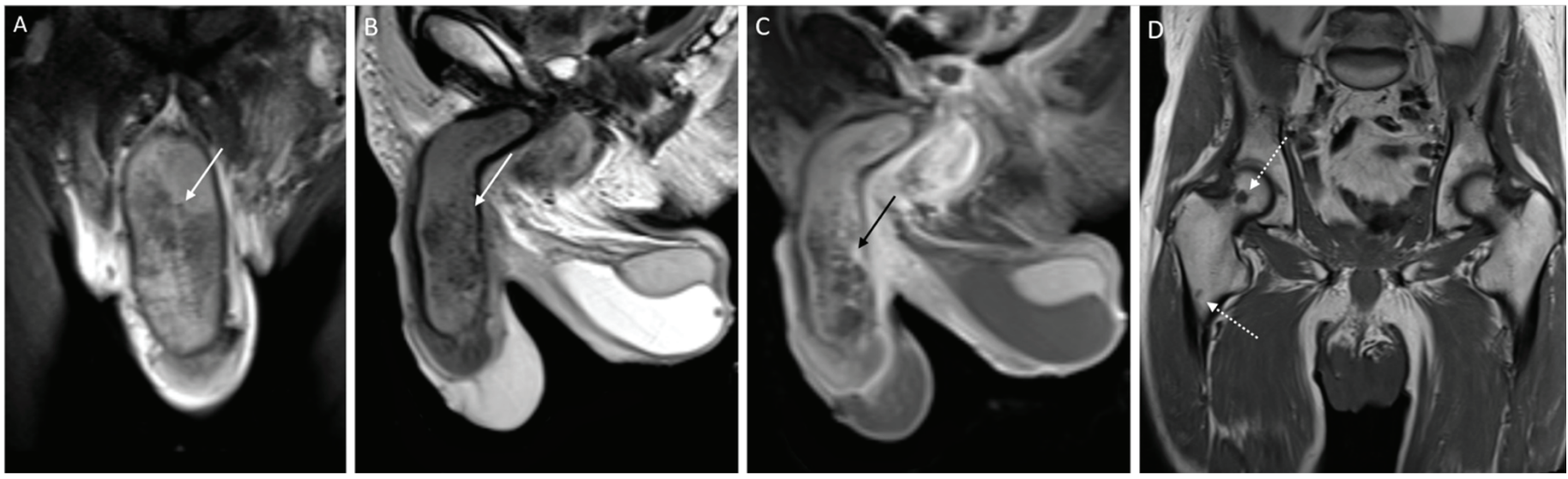

Figure 1. Penile magnetic resonance. Coronal and Sagittal T2-weighted images $(A, B)$ showing patchy ill-defined areas of decreased signal intensity along both corpora cavernosa (white arrows). Diffuse thickening and increased signal intensity of the skin and the areolar tissue are also seen, which reflect edema. Sagittal T1-weighted image after the administration of gadolinium (C) revealing hypoenhancing areas in the mid and distal portions of the left corpus cavernosum (black arrow), consistent with hypovascularity due to tumoral invasion with associated microthrombosis. A moderate-size left hydrocele is also evident. (D) coronal T1-weighted image depicts two hypointense nodules in the right femur (dotted arrows), which is suspicious for bone metastases 
benefit from trials of chemotherapy and if the tumor expresses programmed cell death ligand 1, as in this case report, the patient can be allocated to an ongoing multicentric clinical trial with pembrolizumab.

Secondary involvement of the penis typically indicates systemic dissemination and, consequently, a poor prognosis. A recent systematic review by Cocci et al. (3), based on published cases, found a mean survival time of 14.5 months for these patients.

\section{Ethics}

Informed Consent: Written informed patient consent for publication has been obtained.

Peer-review: Internally peer-reviewed.

\section{Authorship Contributions}

Surgical and Medical Practices: S.C.D., P.A.M., D.S., R.P., Concept: M.C.D.S., A.T.V., S.C.D., R.P., A.J.M., Design: M.C.D.S., A.T.V., D.S., A.J.M., Data Collection or Processing: M.C.D.S., S.C.D., R.P., Analysis or Interpretation: M.C.D.S., S.C.D., R.P., Literature Search: M.C.D.S., D.S., Writing: M.C.D.S., A.T.V., S.C.D., P.A.M., D.S., R.P., A.J.M.
Conflict of Interest: No conflict of interest was declared by the authors.

Financial Disclosure: The authors declared that this study received no financial support.

\section{References}

1. Eberth C. Krebsmetastasen des corpus cavernosum penis. Virchows Archiv 1870;51:145-146.

2. Zhang $K$, Da J, Yao HJ, Zheng DC, Cai ZK, Jiang YQ, Xu MX, Wang Z. Metastatic tumors of the penis: a report of 8 cases and review of the literature. Medicine (Baltimore) 2015;94:e132.

3. Cocci A, Hakenberg OW, Cai T, Nesi G, Livi L, Detti B, Minervini A, Morelli G, Carini M, Serni S, Gacci M. Prognosis of men with penile metastasis and malignant priapism: a systematic review. Oncotarget 2017;9:2923-2930.

4. Mearini L, Colella R, Zucchi A, Nunzi E, Porrozzi C, Porena M. A review of penile metastasis. Oncol Rev 2012;6:e10.

5. Triki W, Kacem A, Itami A, Baraket O, Rebai MH, Bouchoucha S. Penile metastasis of colon carcinoma: A rare case report. Urol Case Rep 2019;24:100875

6. Chaux A, Amin M, Cubilla AL, Young RH. Metastatic tumors to the penis: a report of 17 cases and review of the literature. Int J Surg Pathol $2011 ; 19: 597-606$ 